\title{
The generalized 3-connectivity of Cartesian product graphs $\|^{\dagger}$
}

\author{
Hengzhe Li" Xueliang Li Yuefang Sun \\ Center for Combinatorics and LPMC-TJKLC, Nankai University, Tianjin, China \\ received $4^{\text {th }}$ May 2011, revised 20 $0^{\text {th }}$ November 2011, $11^{\text {th }}$ January 2012, , accepted $19^{\text {th }}$ February 2012.
}

The generalized connectivity of a graph, which was introduced by Chartrand et al. in 1984, is a generalization of the concept of vertex connectivity. Let $S$ be a nonempty set of vertices of $G$, a collection $\left\{T_{1}, T_{2}, \ldots, T_{r}\right\}$ of trees in $G$ is said to be internally disjoint trees connecting $S$ if $E\left(T_{i}\right) \cap E\left(T_{j}\right)=\emptyset$ and $V\left(T_{i}\right) \cap V\left(T_{j}\right)=S$ for any pair of distinct integers $i, j$, where $1 \leq i, j \leq r$. For an integer $k$ with $2 \leq k \leq n$, the $k$-connectivity $\kappa_{k}(G)$ of $G$ is the greatest positive integer $r$ for which $G$ contains at least $r$ internally disjoint trees connecting $S$ for any set $S$ of $k$ vertices of $G$. Obviously, $\kappa_{2}(G)=\kappa(G)$ is the connectivity of $G$. Sabidussi's Theorem showed that $\kappa(G \square H) \geq \kappa(G)+\kappa(H)$ for any two connected graphs $G$ and $H$. In this paper, we prove that for any two connected graphs $G$ and $H$ with $\kappa_{3}(G) \geq \kappa_{3}(H)$, if $\kappa(G)>\kappa_{3}(G)$, then $\kappa_{3}(G \square H) \geq \kappa_{3}(G)+\kappa_{3}(H)$; if $\kappa(G)=\kappa_{3}(G)$, then $\kappa_{3}(G \square H) \geq \kappa_{3}(G)+\kappa_{3}(H)-1$. Our result could be seen as an extension of Sabidussi's Theorem. Moreover, all the bounds are sharp.

Keywords: Connectivity, Generalized connectivity, Internally disjoint path, Internally disjoint trees.

\section{Introduction}

All graphs in this paper are undirected, finite and simple. We refer to the book [1] for graph theoretic notations and terminologies not described here. For any graph $G$, the connectivity $\kappa(G)$ of a graph $G$ is defined as $\min \{|S|: S \subseteq V(G)$ and $G-S$ is disconnected or trivial $\}$. Whitney [14] showed an equivalent definition of the connectivity of a graph. For each pair of vertices $x, y$ of $G$, let $\kappa(x, y)$ denote the maximum number of internally disjoint paths connecting $x$ and $y$ in $G$. Then the connectivity $\kappa(G)$ of $G$ is $\min \{\kappa(x, y): x, y$ are distinct vertices of $G\}$.

The Cartesian product of graphs is an important method to construct a bigger graph, and plays a key role in design and analysis of networks. In the past several decades, many authors have studied the (edge) connectivity of the Cartesian product graphs. Specially, Sabidussi in [11] derived the following prefect and well-known theorem on the connectivity of Cartesian product graphs.

\footnotetext{
${ }^{\dagger}$ Supported by NSFC No. 11071130.

‡Email: lhz2010@mail.nankai.edu.cn

1365-8050 @ 2012 Discrete Mathematics and Theoretical Computer Science (DMTCS), Nancy, France
} 
Theorem 1.1 (Sabidussi's Theorem [11]) Let $G$ and $H$ be two connected graphs. Then

$$
\kappa(G \square H) \geq \kappa(G)+\kappa(H) .
$$

More information about the (edge) connectivity of the Cartesian product graphs can be found in [4, 5, 6, 11, 12, 15, 16].

The generalized connectivity of a graph $G$, which was introduced by Chartrand et al. in [2], is a natural and nice generalization of the concept of vertex connectivity. A tree $T$ is called an $S$-tree $\left(\left\{u_{1}, u_{2}, \ldots, u_{k}\right\}\right.$-tree $)$ if $S \subseteq V(T)$, where $S=\left\{u_{1}, u_{2}, \ldots, u_{k}\right\} \in V(G)$. A family of trees $T_{1}, T_{2}, \ldots$, $T_{r}$ are internally disjoint $S$-trees if $E\left(T_{i}\right) \cap E\left(T_{j}\right)=\emptyset$ and $V\left(T_{i}\right) \cap V\left(T_{j}\right)=S$ for any pair of integers $i$ and $j$, where $1 \leq i<j \leq r$. We use $\kappa(S)$ to denote the greatest number of internally disjoint $S$-trees. For an integer $k$ with $2 \leq k \leq n$, the $k$-connectivity $\kappa_{k}(G)$ of $G$ is defined as $\min \{\kappa(S) \mid S \in V(G)$ and $|S|=k\}$. Clearly, when $|S|=2, \kappa_{2}(G)$ is nothing new but the connectivity $\kappa(G)$ of $G$, that is, $\kappa_{2}(G)=\kappa(G)$, which is the reason why one addresses $\kappa_{k}(G)$ as the generalized connectivity of $G$. By convention, for a connected graph $G$ with less than $k$ vertices, we set $\kappa_{k}(G)=1$. For any graph $G$, clearly, $\kappa(G) \geq 1$ if and only if $\kappa_{k}(G) \geq 1$.

In addition to being a natural combinatorial measure, the generalized connectivity can be motivated by its interesting interpretation in practice. For example, suppose that $G$ represents a network. If one considers to connect a pair of vertices of $G$, then a path is used to connect them. However, if one wants to connect a set $S$ of vertices of $G$ with $|S| \geq 3$, then a tree has to be used to connect them. This kind of tree with minimum order for connecting a set of vertices is usually called a Steiner tree, and popularly used in the physical design of VLSI, see [13]. Usually, one wants to consider how tough a network can be, for the connection of a set of vertices. Then, the number of totally independent ways to connect them is a measure for this purpose. The generalized $k$-connectivity can serve for measuring the capability of a network $G$ to connect any $k$ vertices in $G$.

In [8], $\mathrm{Li}$ and $\mathrm{Li}$ investigated the complexity of determining the generalized connectivity and derived that for any fixed integer $k \geq 2$, given a graph $G$ and a subset $S$ of $V(G)$, deciding whether there are $k$ internally disjoint trees connecting $S$, namely deciding whether $\kappa(S) \geq k$, is NP-complete. The generalized connectivity of complete bipartite graphs was studied by Okamoto and Zhang in [10], and Li and $\mathrm{Li}$ in [7].

Chartrand et al. [3] got the following result for complete graphs.

Theorem 1.2 [3] For every two integers $n$ and $k$ with $2 \leq k \leq n, \kappa_{k}\left(K_{n}\right)=n-\lceil k / 2\rceil$.

Theorem 1.3 [9] Let $G$ be a connected graph with at least three vertices. If $G$ has two adjacent vertices with minimum degree $\delta$, then $\kappa_{3}(G) \leq \delta-1$.

Theorem 1.4 [9] For any connected graph $G, \kappa_{3}(G) \leq \kappa(G)$. Moreover, the upper bound is sharp.

In this paper, we study the 3-connectivity of Cartesian product graphs and get the following result.

Theorem 1.5 Let $G$ and $H$ be connected graphs such that $\kappa_{3}(G) \geq \kappa_{3}(H)$. The following assertions hold:

(i) If $\kappa(G)=\kappa_{3}(G)$, then $\kappa_{3}(G \square H) \geq \kappa_{3}(G)+\kappa_{3}(H)-1$. Moreover, the bound is sharp;

(ii) If $\kappa(G)>\kappa_{3}(G)$, then $\kappa_{3}(G \square H) \geq \kappa_{3}(G)+\kappa_{3}(H)$. Moreover, the bound is sharp. 
The paper is organized as follows. In Section 2, we recall the definition and properties of Cartesian product graphs, and give some basic results about the internally disjoint $S$-trees. As usual, in order to get a general result, we begin with a special case. In Section 3 , we study the 3-connectivity of the Cartesian product of a graph $G$ and a tree $T$. This section is a preparation of Section 4 In Section 4 we study the 3 -connectivity of the Cartesian product of two connected graphs $G$ and $H$. Moreover, all the bounds are sharp. Our result could be seen as an extension of Theorem 1.1

\section{Some basic results}

We use $P_{n}$ to denote a path with $n$ vertices. A path $P$ is called a $u$-v path, denoted by $P_{u, v}$, if $u$ and $v$ are the endpoints of $P$.

Recall that the Cartesian product (also called the square product) of two graphs $G$ and $H$, written as $G \square H$, is the graph with vertex set $V(G) \times V(H)$, in which two vertices $(u, v)$ and $\left(u^{\prime}, v^{\prime}\right)$ are adjacent if and only if $u=u^{\prime}$ and $\left(v, v^{\prime}\right) \in E(H)$, or $v=v^{\prime}$ and $\left(u, u^{\prime}\right) \in E(G)$. Clearly, the Cartesian product is commutative, that is, $G \square H \cong H \square G$. The edge $(u, v)\left(u^{\prime}, v^{\prime}\right)$ is called one-type edge if $\left(u, u^{\prime}\right) \in E(G)$ and $v=v^{\prime}$; similarly, the $(u, v)\left(u^{\prime}, v^{\prime}\right)$ is called two-type edge if $u=u^{\prime}$ and $\left(v, v^{\prime}\right) \in E(H)$.

Let $G$ and $H$ be two graphs with $V(G)=\left\{u_{1}, u_{2}, \ldots, u_{n}\right\}$ and $V(H)=\left\{v_{1}, v_{2}, \ldots, v_{m}\right\}$, respectively. We use $G\left(u_{j}, v_{i}\right)$ to denote the subgraph of $G \square H$ induced by the set $\left\{\left(u_{j}, v_{i}\right) \mid 1 \leq j \leq n\right\}$. Similarly, we use $H\left(u_{j}, v_{i}\right)$ to denote the subgraph of $G \square H$ induced by the set $\left\{\left(u_{j}, v_{i}\right) \mid 1 \leq i \leq m\right\}$. It is easy to see $G\left(u_{j_{1}}, v_{i}\right)=G\left(u_{j_{2}}, v_{i}\right)$ for different $u_{j_{1}}$ and $u_{j_{2}}$ of $G$. Thus, we can replace $G\left(u_{j}, v_{i}\right)$ by $G\left(v_{i}\right)$ for simplicity. Similarly, we can replace $H\left(u_{j}, v_{i}\right)$ by $H\left(u_{j}\right)$. For any $u, u^{\prime} \in V(G)$ and $v, v^{\prime} \in V(G),(u, v),\left(u, v^{\prime}\right) \in V(H(u)),\left(u^{\prime}, v\right),\left(u^{\prime}, v^{\prime}\right) \in V\left(H\left(u^{\prime}\right)\right),(u, v),\left(u^{\prime}, v\right) \in V(G(v))$, and $\left(u, v^{\prime}\right),\left(u^{\prime}, v^{\prime}\right) \in V(G(v))$. We refer to $\left(u, v^{\prime}\right)$ and $\left(u^{\prime}, v\right)$ as the vertices corresponding to $(u, v)$ in $G\left(v^{\prime}\right)\left(=G\left(u, v^{\prime}\right)\right)$ and $H\left(u^{\prime}\right)\left(=H\left(u^{\prime}, v\right)\right)$, respectively. Similarly, we can define the path and tree corresponding to some path and tree, respectively.

In order to show our main results, we need the following well-known theorem.

Theorem 2.1 (Menger's Theorem [1]) Let $G$ be a k-connected graph, and let $x$ and $y$ be a pair of distinct vertices in $G$. Then there exist $k$ internally disjoint paths $P_{1}, P_{2}, \ldots, P_{k}$ in $G$ connecting $x$ and $y$.

Let $G$ be a connected graph, and $S=\left\{x_{1}, x_{2}, x_{3}\right\} \subseteq V(G)$. We first have the following observation about internally disjoint $S$-trees.

Observation 2.1 Let $G$ be a connected graph, $S=\left\{x_{1}, x_{2}, x_{3}\right\} \subseteq V(G)$, and $T$ be an $S$-tree. Then there exists a subtree $T^{\prime}$ of $T$ such that $T^{\prime}$ is also an $S$-tree such that $1 \leq d_{T^{\prime}}\left(x_{i}\right) \leq 2, \mid\left\{x_{i} \mid d_{T^{\prime}}\left(x_{i}\right)=\right.$ $1\} \mid \geq 2$ and $\left\{x \mid d_{T^{\prime}}(x)=1\right\} \subseteq S$. Moreover, if $\left|\left\{x_{i} \mid d_{T^{\prime}}\left(x_{i}\right)=1\right\}\right|=3$, then all the vertices of $V\left(T^{\prime}\right) \backslash\left\{x_{1}, x_{2}, x_{3}\right\}$ have degree 2 except for one vertex, say $x$ with $d_{T^{\prime}}(x)=3$; if there exists one vertex of $S$, say $x_{1}$, of degree 2 in $T^{\prime}$, then $T^{\prime}$ is an $x_{2}-x_{3}$ path.

Proof: It is easy to check that this observation holds by deleting vertices and edges of $T$.

Remark 2.1 (i) Since the path between any two distinct vertices is unique in $T$, the tree $T^{\prime}$ obtained from $T$ in Observation 2.1 is unique. Such a tree is called a minimal $S$-tree (or minimal $\left\{x_{1}, x_{2}, x_{3}\right\}$-tree).

(ii) Let $S=\{x, y, z\} \subseteq V(G)$. Throughout this paper, we can assume that each $S$-tree is a minimal $S$-tree. 
Lemma 2.1 Let $G$ be a graph with $\kappa_{3}(G)=k \geq 2, S=\{x, y, z\} \subseteq V(G)$. Then, the following assertions hold:

(i) If $G[S]$ is a clique, then there exist $k$ internally disjoint $S$-trees $T_{1}, T_{2}, \ldots, T_{k}$, such that $E\left(T_{i}\right) \cap$ $E(G[S])=\emptyset$ for $1 \leq i \leq k-2$.

(ii) If $G[S]$ is not a clique, then there exist $k$ internally disjoint $S$-trees $T_{1}, T_{2}, \ldots, T_{k}$, such that $E\left(T_{i}\right) \cap E(G[S])=\emptyset$ for $1 \leq i \leq k-1$.

Proof: We first prove (i). Clearly, by the definition of $S$-trees, we know $\left|\left\{T_{i} \mid E\left(T_{i}\right) \cap E(G[S]) \neq \emptyset\right\}\right| \leq$ 3. Let $\left\{T_{1}, T_{2}, \ldots, T_{k}\right\}$ be $k$ internally disjoint $S$-trees. If $\left|\left\{T_{i} \mid E\left(T_{i}\right) \cap E(G[S]) \neq \emptyset\right\}\right| \leq 2$, we are done by exchanging subscript. Thus, suppose $\left|\left\{T_{i} \mid E\left(T_{i}\right) \cap E(G[S]) \neq \emptyset\right\}\right|=3$. Without loss of generality, we assume $E\left(T_{i}\right) \cap E(G[S]) \neq \emptyset$, where $i=k-2, k-1, k$. It is easy to check that $T_{k-2}, T_{k-1}, T_{k}$ must have the structures as shown in Figures $1 a$ and $c$. But, for these two cases, we can obtain $T_{k-2}^{\prime}, T_{k-1}^{\prime}, T_{k}^{\prime}$ from $T_{k-2}, T_{k-1}, T_{k}$, such that $E\left(T_{k-2}^{\prime}\right) \cap\{x y, x z, y z\}=\emptyset$. See Figs. $1 b$. and $1 d$, where the tree $T_{k-2}^{\prime}$ is shown by dotted lines. Thus $T_{1}, T_{2}, \ldots, T_{k-3}, T_{k-2}^{\prime}, T_{k-1}^{\prime}, T_{k}^{\prime}$ are our desired $S$-trees.

The proof of $(i i)$ is similar to that of $(i)$, and thus is omitted.

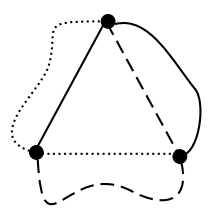

Fig. 1a.

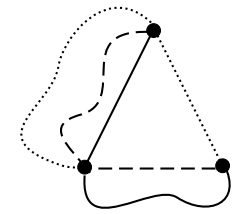

Fig. 1c.

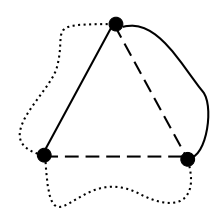

Fig. $1 b$.

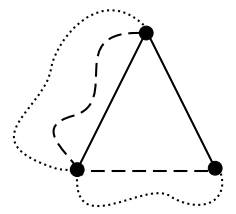

Fig. 1d.

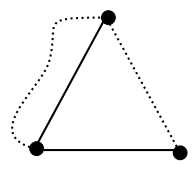

Fig. 1e.

Fig. 1: $T_{k-2}^{\prime}, T_{k-1}^{\prime}, T_{k}^{\prime}$. An edge is shown by a straight line. The edges (or paths) of a tree are shown by the same type of lines.

Remark 2.2 Let $G$ be a graph with $\kappa_{3}(G)=k \geq 2, S=\{x, y, z\} \subseteq V(G)$. If $\mid\left\{T_{i} \mid E\left(T_{i}\right) \cap E(G[S]) \neq\right.$ $\emptyset\} \mid \geq 2$ for any collection $\mathcal{T}$ of $k$ internally disjoint $S$-trees, then $G[S]$ is a clique. Moreover, $T_{k-1} \cup T_{k}$ must have the structure as shown in Figure 17 . 


\section{The Cartesian product of a connected graph and a path}

In this section, we show the following proposition.

Proposition 3.1 Let $G$ be a graph and $P_{m}$ be a path with $m$ vertices. The following assertions hold:

(i) If $\kappa_{3}(G)=\kappa(G) \geq 1$, then $\kappa_{3}\left(G \square P_{m}\right) \geq \kappa_{3}(G)$. Moreover, the bound is sharp;

(ii) If $1 \leq \kappa_{3}(G)<\kappa(G)$, then $\kappa_{3}\left(G \square P_{m}\right) \geq \kappa_{3}(G)+1$. Moreover, the bound is sharp.

We shall prove Proposition 3.1 by a series of lemmas. Since the proofs of $(i)$ and $(i i)$ are similar, we only show $(i i)$. Let $G$ be a graph with $V(G)=\left\{u_{1}, u_{2}, \ldots, u_{n}\right\}$ such that $1 \leq \kappa_{3}(G)<\kappa(G)$, $V\left(P_{m}\right)=\left\{v_{1}, v_{2}, \ldots, v_{m}\right\}$ such that $v_{i}$ and $v_{j}$ are adjacent if and only if $|i-j|=1$.

Set $\kappa_{3}(G)=k$ for simplicity. To prove $(i i)$, we need to show that for any $S=\{x, y, z\} \subseteq V(G \square H)$, there exist $k+1$ internally disjoint $S$-trees. We proceed our proof by the following three lemmas.

Lemma 3.1 If $x, y, z$ belongs to the same $V\left(G\left(v_{i}\right)\right), 1 \leq i \leq m$, then there exist $k+1$ internally disjoint S-trees.

Proof: Without loss of generality, we assume $x, y, z \in V\left(G\left(v_{1}\right)\right)$. Since $\kappa_{3}(G)=k$, there exist $k$ internally disjoint $S$-trees $T_{1}, T_{2}, \ldots, T_{k}$ in $G\left(v_{1}\right)$. We need another $S$-tree $T_{k+1}$ such that $T_{k+1}$ and $T_{i}$ are internally disjoint, for $i=1,2, \ldots, k$. Let $x^{\prime}, y^{\prime}, z^{\prime}$ be the vertices corresponding to $x, y, z$ in $G\left(v_{2}\right)$, and $T_{1}^{\prime}$ be the tree corresponding to $T_{1}$ in $G\left(v_{2}\right)$. Therefore, tree $T_{k+1}$ obtained from $T_{1}^{\prime}$ by adding three edges $x x^{\prime}, y y^{\prime}, z z^{\prime}$ is a desired tree.

Lemma 3.2 If only two vertices of $\{x, y, z\}$ belong to some copy $G\left(v_{i}\right)$, then there exist $k+1$ internally disjoint $S$-trees.

Proof: We may assume $x, y \in V\left(G\left(v_{1}\right)\right), z \in V\left(G\left(v_{2}\right)\right)$. In the following argument, we can see that this assumption has no influence on the correctness of our proof. Let $x^{\prime}, y^{\prime}$ be the vertices corresponding to $x, y$ in $G\left(v_{2}\right), z^{\prime}$ be the vertex corresponding to $z$ in $G\left(v_{1}\right)$. Consider the following two cases.

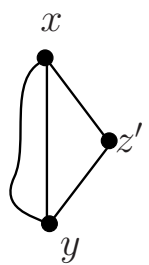

$2 a$

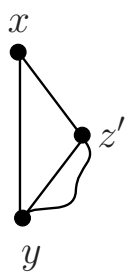

$2 b$

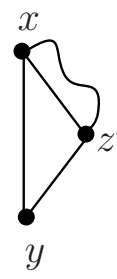

$2 c$

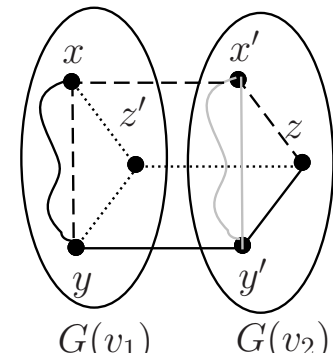

$2 d$

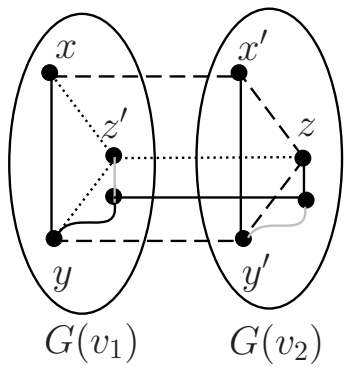

$2 e$

Fig. 2: The edges (or paths) of a tree are shown by the same type of lines. The lightest lines stand for edges (or paths) not contained in $T_{i}^{*}$. 
Case 1: $z^{\prime} \notin\{x, y\}$.

Let $S^{\prime}=\left\{x, y, z^{\prime}\right\}$, and $T_{1}, T_{2}, \ldots T_{k}$ be $k$ internally disjoint $S^{\prime}$-trees in $G\left(v_{1}\right)$ such that $\mid\left\{T_{i}\left|E\left(T_{i}\right) \cap E\left(G\left(v_{1}\right)\left[S^{\prime}\right]\right\} \neq \emptyset\right|\right.$ is as small as possible. We can assume that $E\left(T_{i}\right) \cap E\left(G\left(v_{1}\right)\left[S^{\prime}\right]\right)=$ $\emptyset$ for each $i$, where $1 \leq i \leq k-2$ by Lemma 2.1.

For a tree $T_{i}$ with $E\left(T_{i}\right) \cap E\left(G\left(v_{1}\right)\left[S^{\prime}\right]\right)=\emptyset$, let $T_{i}^{*}$ be the tree obtained from $T_{i}$ by adding $z_{i} z_{i}^{\prime}$ and $z_{i}^{\prime} z$, and deleting $z^{\prime}$, where $z_{i}$ is any one neighbor of $z^{\prime}$ in $T_{i}$, and $z_{i}^{\prime}$ is the vertex corresponding to $z_{i}$ in $G\left(v_{2}\right)$.

If $E\left(T_{k}\right) \cap E\left(G\left(v_{1}\right)\left[S^{\prime}\right]\right) \neq \emptyset$, say $y z^{\prime} \in E\left(T_{k}\right) \cap E\left(G\left(v_{1}\right)\left[S^{\prime}\right]\right)$. Let $T_{k}^{*}=T_{k}+z z^{\prime}$ and $T_{k+1}^{*}=T_{k}^{\prime}+x x^{\prime}+y y^{\prime}$, where $T_{k}^{\prime}$ is the tree corresponding to $T_{k}$ in $G\left(v_{2}\right)$.

If $E\left(T_{k-1}\right) \cap E\left(G\left(v_{1}\right)\left[S^{\prime}\right]\right) \neq \emptyset$ and $E\left(T_{k}\right) \cap E\left(G\left(v_{1}\right)\left[S^{\prime}\right]\right) \neq \emptyset$. Then $T_{k-1} \cup T_{k}$ must have one of the structures as shown in Figures $2 \mathrm{a}, \mathrm{b}$ and $\mathrm{c}$ by Remark 2.2. If $T_{k-1}$ and $T_{k}$ have the structures as shown in Figure 2 a, then we can obtain trees $T_{k-1}^{*}, T_{k}^{*}$ and $T_{k+1}^{*}$ as shown in Figure 2 . If $T_{k-1}$ and $T_{k}$ have the structures as shown in Figure 2 , then we can obtain trees $T_{k-1}^{*}, T_{k}^{*}$ and $T_{k+1}^{*}$ as shown in Figure 2 . If $T_{k-1}$ and $T_{k}$ have the structures as shown in Figure 2 , then we can obtain trees $T_{k-1}^{*}, T_{k}^{*}$ and $T_{k+1}^{*}$ similar to those in Figure $2 \mathrm{~d}$.

Case 2: $z^{\prime} \in\{x, y\}$.

Without loss of generality, assume $z^{\prime}=y$. Since $\kappa(G)>\kappa_{3}(G)=k$, by Menger's Theorem, there exist at least $k+1$ internally disjoint $x-y$ paths $P^{1}, P^{2}, \ldots, P^{k+1}$ in $G\left(v_{1}\right)$. Assume that $y_{i}$ is the only neighbor of $y$ in $P^{i}$, and that $y_{i}^{\prime}$ is the vertex corresponding to $y_{i}$ in $G\left(v_{2}\right)$. If $x$ and $y$ are nonadjacent in $P^{i}$, let $T_{i}$ be the tree obtained from $P^{i}$ by adding $y_{i} y_{i}^{\prime}$ and $y_{i}^{\prime} z$. If $x$ and $y$ are adjacent in $P^{i}$, let $T_{i}$ be the tree obtained from $P^{i}$ by adding $y z$. Since $G$ is a simple graph, there exists at most one path $P^{i}$ such that $x$ and $y$ are adjacent on $P^{i}$. Thus $T_{i}, 1 \leq i \leq k+1$, are $k+1$ internally disjoint $S$-trees.

Lemma 3.3 If $x, y, z$ are contained in distinct $G\left(v_{i}\right) s$, then there exist $k+1$ internally disjoint $S$-trees.

Proof: We may assume that $x \in V\left(G\left(v_{1}\right)\right), y \in V\left(G\left(v_{2}\right)\right), z \in V\left(G\left(v_{3}\right)\right)$. In the following argument, we can see that this assumption has no influence on the correctness of our proof. Let $y^{\prime}, z^{\prime}$ be the vertices corresponding to $y, z$ in $G\left(v_{1}\right), x^{\prime}, z^{\prime \prime}$ be the vertices corresponding to $x, z$ in $G\left(v_{2}\right)$ and $x^{\prime \prime}, y^{\prime \prime}$ be the vertices corresponding to $x, y$ in $G\left(v_{3}\right)$. We consider the following three cases.

Case 1: $x, y^{\prime}, z^{\prime}$ are distinct vertices in $G\left(v_{1}\right)$

Let $S^{\prime}=\left\{x, y^{\prime}, z^{\prime}\right\}$, and $T_{1}, T_{2}, \ldots T_{k}$ be $k$ internally disjoint $S^{\prime}$-trees in $G\left(v_{1}\right)$ such that $\left|\left\{T_{i} \mid E\left(T_{i}\right) \cap E\left(G\left(v_{1}\right)\left[S^{\prime}\right]\right) \neq \emptyset\right\}\right|$ is as small as possible. We can assume that $E\left(T_{i}\right) \cap$ $E\left(G\left(v_{1}\right)\left[S^{\prime}\right]\right)=\emptyset$ for each $i$, where $1 \leq i \leq k-2$ by Lemma 2.1. For each $T_{i}$ such that $E\left(T_{i}\right) \cap E\left(G\left(v_{1}\right)\left[S^{\prime}\right]\right)=\emptyset$, we can obtain an $S$-tree $T_{i}^{*}$ from $T_{i}$ similar to that in Subcase 1.1 of Lemma 3.2

If $E\left(T_{k-1}\right) \cap E\left(G\left(v_{1}\right)\left[S^{\prime}\right]\right)=\emptyset$ or $E\left(T_{k-1}\right) \cap E\left(G\left(v_{1}\right)\left[S^{\prime}\right]\right)=\emptyset$. Without loss of generality, we assume $E\left(T_{k-1}\right) \cap E\left(G\left(v_{1}\right)\left[S^{\prime}\right]\right)=\emptyset$. Let $T_{k}^{*}$ be the tree obtained from $T_{k}$ by adding edges 
The generalized 3-connectivity of Cartesian product graphs

$y^{\prime} y, z^{\prime} z^{\prime \prime}$ and $z^{\prime \prime} z, T_{k+1}^{*}$ be the tree obtained from $T_{k}^{\prime \prime}$ by adding $x^{\prime \prime} x^{\prime}, x^{\prime} x$ and $y^{\prime \prime} y$, where $T_{k}^{\prime \prime}$ is the tree corresponding to $T_{k}$ in $G\left(v_{3}\right)$. Thus, $T_{i}^{*} \mathrm{~s}, 1 \leq i \leq k+1$, are $k$ internally disjoint $S$-tree.

Otherwise, that is, $E\left(T_{k-1}\right) \cap E\left(G\left(v_{1}\right)\left[S^{\prime}\right]\right) \neq \emptyset$ and $E\left(T_{k}\right) \cap E\left(G\left(v_{1}\right)\left[S^{\prime}\right]\right) \neq \emptyset$. Then $T_{k-1}$ and $T_{k}$ must have the structures as shown in Figure $3 \mathrm{k}, \mathrm{b}$ and c. If $T_{k-1}$ and $T_{k}$ have the structures as shown in Figure 3 a, then we can obtain trees $T_{k-1}^{*}, T_{k}^{*}$ and $T_{k+1}^{*}$ as shown in Figure $3 \mathrm{~d}$. If $T_{k-1}$ and $T_{k}$ have the structures as shown in Figure $3 \mathrm{p}$, then we can obtain trees $T_{k-1}^{*}, T_{k}^{*}$ and $T_{k+1}^{*}$ as shown in Figure 3 . If $T_{k-1}$ and $T_{k}$ have the structures as shown in Figure $3 k$, then we can obtain trees $T_{k-1}^{*}, T_{k}^{*}$ and $T_{k+1}^{*}$ as shown in Figure 3 .

Case 2: Two of $x, y^{\prime}, z^{\prime}$ are the same vertex in $G\left(v_{1}\right)$.

If $y^{\prime}=z^{\prime}$, since $\kappa(G)>\kappa_{3}(G)=k$, by Menger's Theorem, it is easy to construct $k+1$ internally disjoint $S$-trees. See Figure $3 g$. The other cases $\left(x=y^{\prime}\right.$ or $\left.x=z^{\prime}\right)$ can be proved with similar arguments.

Case 3: $x, y^{\prime}, z^{\prime}$ are the same vertex in $G\left(v_{1}\right)$.

Since $\kappa(G)>\kappa_{3}(G)=k$, by Menger's Theorem, it is easy to construct $k+1$ internally disjoint $S$-trees. See Figure 3 h.

We have the following observation by the argument in the proof of Proposition 3.1 .

Observation 3.1 The $k+1$ internally disjoint S-trees consist of three kinds of edges - the edges of original trees (or paths), the edges corresponding the edges of original trees (or paths) and two-type edges.

Note that $Q_{n} \cong P_{2} \square P_{2} \square \cdots \square P_{2}$, where $Q_{n}$ is the $n$-hypercube. We have the following corollary.

Corollary 3.1 Let $Q_{n}$ be the n-hypercube with $n \geq 2$. Then $\kappa_{3}\left(Q_{n}\right)=n-1$.

Proof: Recall that $\kappa\left(Q_{n}\right)=n$ so that Proposition 3.1 (ii) inductively applies. It is easy to check that $\kappa_{3}\left(Q_{2}\right)=1$. Suppose that the result holds for $Q_{n-1}$, where $n \geq 3$. We have $\kappa_{3}\left(Q_{n}\right) \geq n-1$ by Proposition 3.1. On the other hand, since $Q_{n}$ is $n$-regular, we have $\kappa_{3}\left(Q_{n}\right) \leq n-1$ by Theorem 1.3 . Thus $\kappa_{3}\left(Q_{n}\right)=n-1$.

Example 3.1 Let $K_{2 n}$ be the complete graph with vertex set $V\left(K_{2 n}\right)=\left\{u_{1}, u_{2}, \ldots, u_{2 n}\right\}$, and let $G_{n}$ be the graph obtained from $K_{2 n}$ by adding a new vertex $u$ and edges $u u_{i}, 1 \leq i \leq n$.

For any $S=\{x, y, z\} \subseteq V(G)$, if $u \notin S$, then there exist $k$ internally disjoint $S$-trees in $G_{n}$ by Theorem 1.2. If $u \in S$, without loss of generality, assume $x=u, y=u_{1}, z=u_{2}$. Let $T_{1}$ be the path $u, u_{1}, u_{k+1}, u_{2}, T_{2}$ be the path $u, u_{2}, u_{k+2}, u_{1}$, and $T_{i}$ be the tree obtained from a path $u, u_{n+i}, u_{1}$ by adding an edge $u_{n+i} u_{2}$ for $3 \leq i \leq n$. Clearly, $T_{i}, 1 \leq i \leq n$, are $n$ internally disjoint $S$-trees. So $\kappa_{3}\left(G_{n}\right) \geq n$. Since $\delta\left(G_{n}\right)=n, \kappa_{3}\left(G_{n}\right)=n$ by Theorem 1.4 By Proposition 3.1 $\kappa_{3}\left(G_{n} \square K_{2}\right) \geq n$. Since $G_{n}$ has two adjacent vertices of degree $n+1, \kappa_{3}\left(G_{n}\right)=n$ by Theorem 1.3 Moreover, clearly, $\kappa(G)=n$. Thus $\kappa_{3}\left(G \square K_{2}\right)=\kappa_{3}(G)=n$.

Remark 3.1 We know that the bounds of $(i)$ and $(i i)$ in Theorem 3.1 are sharp by Example 3.1 and Corollary 3.1 

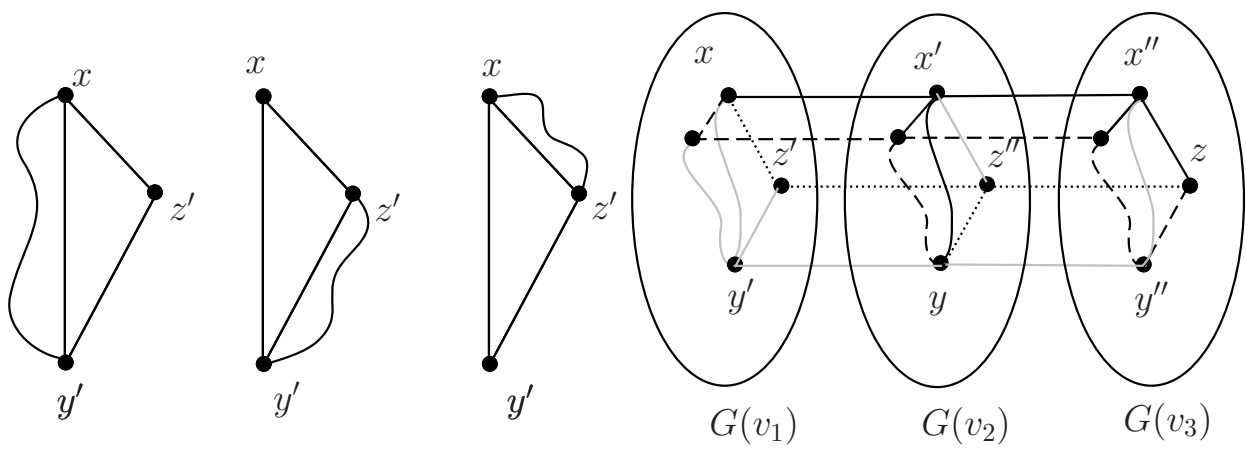

$3 a$

$3 b$

$3 c$

3d. $\quad T_{k}^{*}$ and $T_{k+1}^{*}$
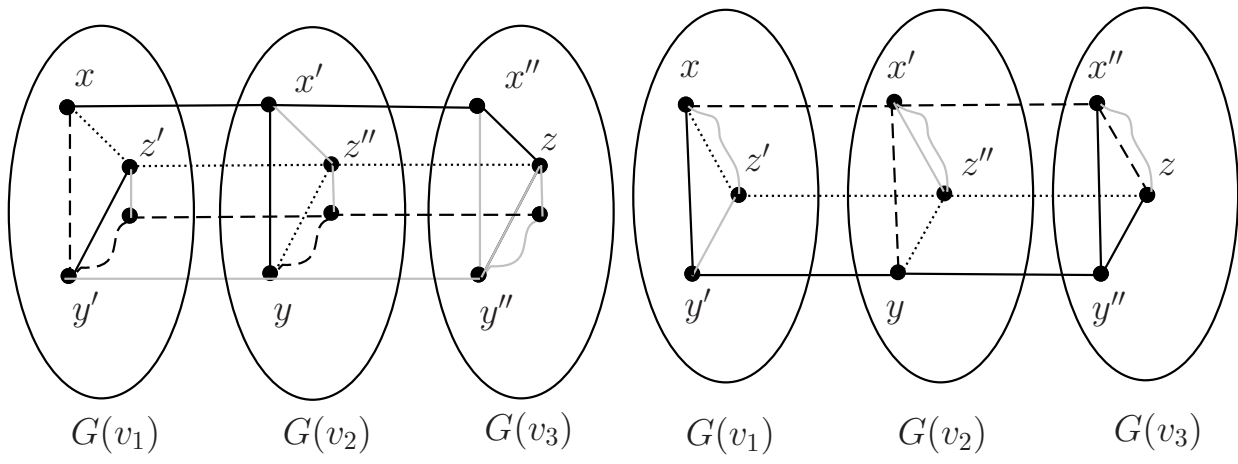

3e $\quad T_{k-1}^{*}, T_{k}^{*}$ and $T_{k+1}^{*}$

3f. $\quad T_{k}^{*}$ and $T_{k+1}^{*}$
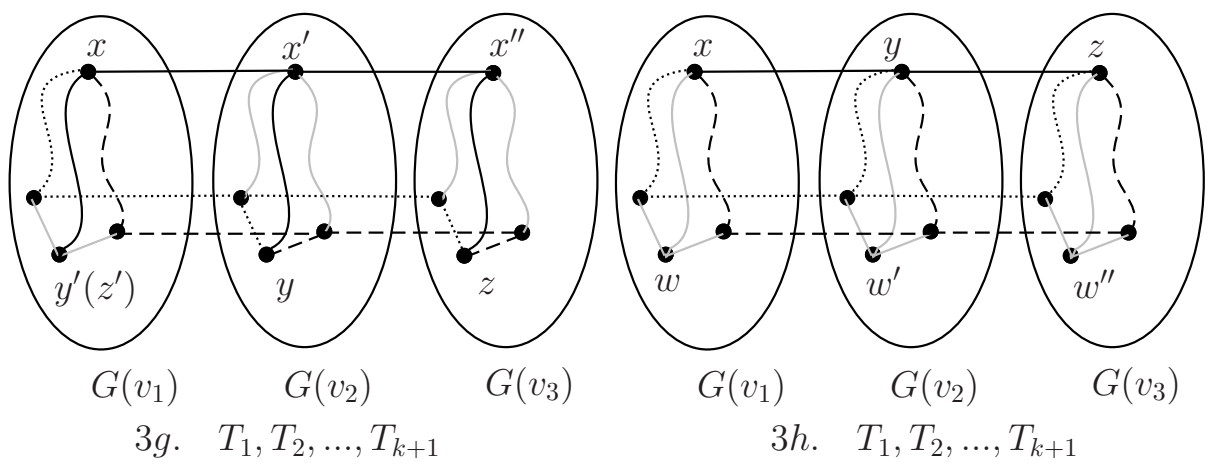

3h. $T_{1}, T_{2}, \ldots, T_{k+1}$

Fig. 3: The edges (or paths) of a tree are shown by the same type of lines. The lightest lines stand for edges (or paths) not contained in $T_{i}^{*}$. 
Proposition 3.2 Let $G$ be a connected graph and $T$ be a tree. The following assertions hold:

(i) If $\kappa_{3}(G)=\kappa(G) \geq 1$, then $\kappa_{3}(G \square T) \geq \kappa_{3}(G)$. Moreover, the bound is sharp;

(ii) If $1 \leq \kappa_{3}(G)<\kappa(G)$, then $\kappa_{3}(G \square T) \geq \kappa_{3}(G)+1$. Moreover, the bound is sharp.

Proof: Since the proofs of $(i)$ and $(i i)$ are similar, we only show $(i i)$. It suffices to show that for any $S=\{x, y, z\} \subseteq V(G \square H)$, there exist $k+1$ internally disjoint $S$-trees. Set $\kappa_{3}(G)=k, V(G)=$ $\left\{u_{1}, u_{2}, \ldots, u_{n}\right\}$, and $V(T)=\left\{v_{1}, v_{2}, \ldots, v_{m}\right\}$.

Let $x \in V\left(G\left(v_{i}\right)\right), y \in V\left(G\left(v_{j}\right)\right), z \in V\left(G\left(v_{k}\right)\right)$ be three distinct vertices. If there exists a path in $T$ containing $v_{i}, v_{j}$ and $v_{k}$, then we are done from Proposition 3.1. If $i, j$ and $k$ are not distinct integers, such a path must exist. Thus, suppose that $i, j$ and $k$ are distinct integers, and that there exists no path containing $v_{i}, v_{j}$ and $v_{k}$. By Observation 2.1, there exists a tree $T^{\prime}$ in $T$ such that $d_{T^{\prime}}\left(v_{i}\right)=d_{T^{\prime}}\left(v_{j}\right)=d_{T^{\prime}}\left(v_{k}\right)=1$ and all the vertices of $V(T) \backslash\left\{v_{i}, v_{j}, v_{k}\right\}$ have degree 2 except for one vertex, say $v_{4}$ with $d_{T}\left(v_{4}\right)=3$. Without loss of generality, we set $i=1, j=2, k=3$. Furthermore, we assume $v_{i} v_{4} \in E\left(T^{\prime}\right)$, where $1 \leq i \leq 3$. In the following argument, we can see that this assumption has no influence on the correctness of our proof.

Let $P$ be the unique path in $T^{\prime}$ connecting $v_{1}$ and $v_{2}$. By Proposition 3.1, we can construct $k+1$ internally disjoint $\left\{x, y, z^{\prime}\right\}$-trees $T_{i}, 1 \leq k+1$, in $G \square P$, where $z^{\prime}$ is the vertex corresponding to $z$ in $G\left(v_{4}\right)$. By a similar method of Proposition 3.1. we can construct $k+1$ internally disjoint $S$-trees in $G \square T$ on the basis of these trees.

Remark 3.2 We know that the bounds of $(i)$ and $($ ii $)$ in Proposition 3.2 are sharp by Example 3.1 and Corollary 3.1

Observation 3.2 The $k+1$ internally disjoint $S$-trees consist of three kinds of edges - the edges of original trees (or paths), the edges corresponding the edges of original trees (or paths) and two-type edges.

\section{The Cartesian product of two general graphs}

Observation 4.1 Let $G$ and $H$ be two connected graphs, $x, y, z$ be three distinct vertices in $H$, and $T_{1}, T_{2}, \ldots, T_{k}$ be $k$ internally disjoint $\{x, y, z\}$-trees in $H$. Then $G \square \bigcup_{i=1}^{k} T_{i}=\bigcup_{i=1}^{k}\left(G \square T_{i}\right)$ has the structure as shown in Figure 4. Moreover, $\left(G \square T_{i}\right) \cap\left(G \square T_{j}\right)=G(x) \cup G(y) \cup G(z)$ for $i \neq j$. In order to show the structure of $G \square \bigcup_{i=1}^{k} T_{i}$ clearly, we take $k$ copies of $G(y)$, and $k$ copies of $G(z)$. Note that, these $k$ copes of $G(y)$ (resp. $G(z)$ ) represent the same graph.

Example 4.1 Let $H$ be the complete graph of order 4. The structure of $G \square\left(T_{1} \cup T_{2}\right)$ is shown in Figure 5 .

Now we are ready to prove Theorem 1.5

Proof of Theorem 1.5: Since the proofs of $(i)$ and $(i i)$ are similar, we only show $(i i)$. Without loss of generality, we set $\kappa_{3}(G):=k, \kappa_{3}(H):=\ell$. It suffices to show that for any $S=\{x, y, z\} \subseteq V(G \square H)$, there exist $k+\ell$ internally disjoint $S$-trees. Assume $V(G)=\left\{u_{1}, u_{2}, \ldots, u_{n}\right\}$ and $V(T)=\left\{v_{1}, v_{2}, \ldots, v_{m}\right\}$.

Let $x \in V\left(G\left(v_{i}\right)\right), y \in V\left(G\left(v_{j}\right)\right), z \in V\left(G\left(v_{k}\right)\right)$ be three distinct vertices in $G \square H$. We will do only the case that $i, j, k$ are distinct integers. Other two possibilities are similar. Without loss of generality, set $i=1, j=2, k=3$. Since $\kappa_{3}(H)=\ell$, there exist $\ell$ internally disjoint $\left\{v_{1}, v_{2}, v_{3}\right\}$-trees $T_{i}, 1 \leq i \leq \ell$, in $H$. We use $G_{i}$ to denote $G \square T_{i}$. By Observation 4.1, we know that $G \square \bigcup_{i=1}^{\ell} T_{i}=\bigcup_{i=1}^{\ell} G_{i}$ and 


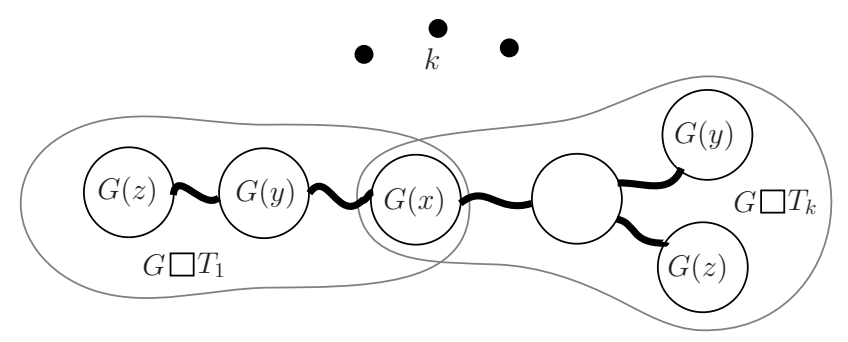

Fig. 4: The structure of $G \square \bigcup_{i=1}^{k} T_{i}$.
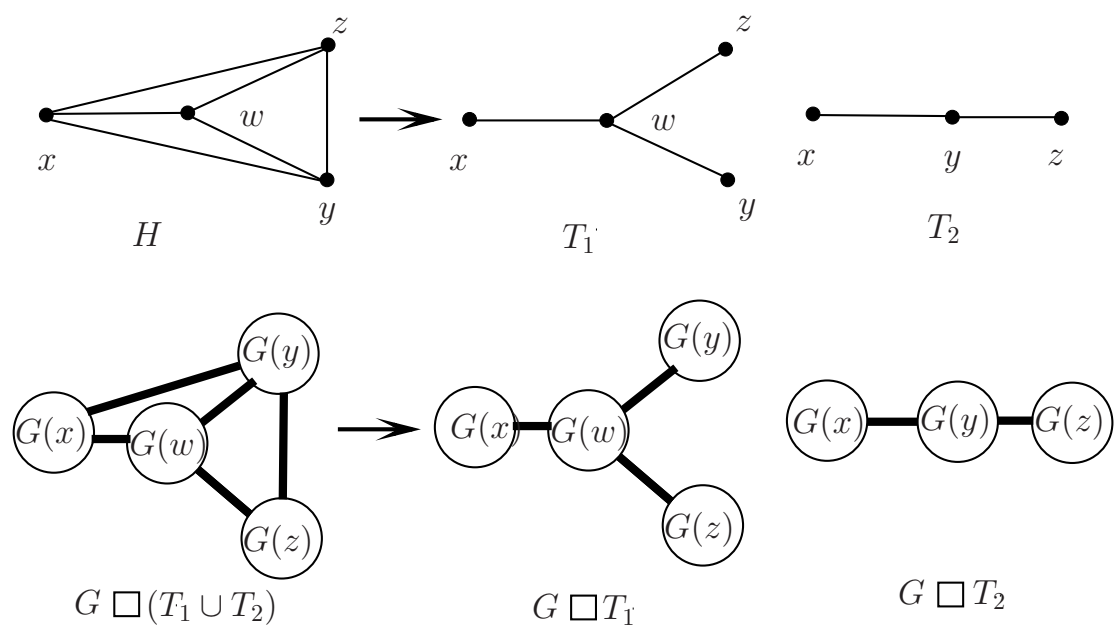

Fig. 5: The structure of $G \square\left(T_{1} \cup T_{2}\right)$. 
$G_{i} \cap G_{j}=G\left(v_{1}\right) \cup G\left(v_{2}\right) \cup G\left(v_{3}\right)$ for $i \neq j$. Let $y^{\prime}, z^{\prime}$ be the vertices corresponding to $y, z$ in $G\left(v_{1}\right)$, respectively.

If $x, y^{\prime}, z^{\prime}$ are distinct vertices in $G\left(v_{1}\right)$. Since $\kappa_{3}\left(G\left(v_{1}\right)\right)=k$, there exist $k$ internally disjoint $\left\{x, y^{\prime}, z^{\prime}\right\}$-trees $T_{j}^{\prime}, 1 \leq j \leq k$, in $G\left(v_{1}\right)$. Let $k_{0}, k_{1}, \ldots, k_{\ell}$ be integers such that $0=k_{0}<k_{1}<\cdots<$ $k_{\ell}=k$. Similar to the proofs of Proposition 3.1, we can construct $k_{i}-k_{i-1}+1$ internally disjoint $S$-trees $T_{i, j_{i}}, 1 \leq j_{i} \leq k_{i}-k_{i-1}+1$, in $\left(\bigcup_{j=k_{i-1}+1}^{k_{i}} T_{j}^{\prime}\right) \square T_{i}$ for each $i$, where $1 \leq i \leq \ell$. By Observations 3.1 and 3.2. $T_{i, j_{i}}$ and $T_{r, j_{r}}$ are internally disjoint for $i \neq r$. Thus $T_{i, j_{i}}, 1 \leq i \leq \ell, 1 \leq j_{i} \leq k_{i}-k_{i-1}+1$ are $k+\ell$ internally disjoint $S$-trees.

If exactly two of $x, y^{\prime}, z^{\prime}$ are the same vertex in $G\left(v_{i}\right)$. Without loss of generality, assume $y^{\prime}=z^{\prime}$. Since $\kappa\left(G\left(v_{1}\right)\right)>k$, there exist $k+1$ internally disjoint $x-y^{\prime}$ paths $P_{i}, 1 \leq i \leq k+1$, in $G\left(v_{1}\right)$ by Menger's Theorem. Note that at most one of them is a path of length 1 . Let $P_{k+1}$ be such a path if $x y^{\prime} \in E\left(G\left(v_{1}\right)\right)$, and let $k_{0}, k_{1}, \ldots, k_{\ell}$ be integers such that $0=k_{0}<k_{1}<\cdots<k_{\ell}=k+1$. Similar to the proofs of Proposition 3.1, we can construct $k_{i}-k_{i-1}+1$ internally disjoint $S$-trees $T_{i, j_{i}}, 1 \leq j_{i} \leq k_{i}-k_{i-1}+1$, in $\left(\bigcup_{j=k_{i-1}+1}^{k_{i}} P_{j}\right) \square T_{i}$ for each $i$, where $1 \leq i \leq \ell-1$, and $k_{\ell}-k_{\ell-1}$ internally disjoint $S$-trees $T_{\ell, j_{\ell}}, 1 \leq j_{i} \leq k_{\ell}-k_{\ell-1}$, in $\left(\bigcup_{j=k_{\ell-1}+1}^{k_{\ell}} P_{j}\right) \square T_{i}$. By Observation 3.1 and 3.2. $T_{i, j_{i}}$ and $T_{r, j_{r}}$ are internally disjoint for $i \neq r$. Thus $T_{i, j_{i}}, 1 \leq i \leq \ell, 1 \leq j_{i} \leq k_{i}-k_{i-1}+1$ are $k+\ell$ internally disjoint $S$-trees.

If all of $x, y^{\prime}, z^{\prime}$ are the same vertex in $G\left(v_{i}\right)$. Since $\delta\left(G\left(v_{1}\right)\right) \geq \kappa\left(G\left(v_{1}\right)\right)>k, x$ has $k$ neighbors, say $x_{1}, x_{2}, \ldots, x_{k}$, in $\left.G\left(v_{1}\right)\right)$. Let $P_{i}$ be the path $x x_{i}$, and let $k_{0}, k_{1}, \ldots, k_{\ell}$ be integers such that $0=k_{0}<$ $k_{1}<\cdots<k_{\ell}=k$. Similar to the proofs of Proposition 3.1, we can construct $k_{i}-k_{i-1}+1$ internally disjoint $S$-trees $T_{i, j_{i}}, 1 \leq j_{i} \leq k_{i}-k_{i-1}+1$, in $\left(\bigcup_{j=k_{i-1}+1}^{k_{i}} P_{j}\right) \square T_{i}$ for each $i$, where $1 \leq i \leq \ell$. By Observation 3.1 and $3.2, T_{i, j_{i}}$ and $T_{r, j_{r}}$ are internally disjoint for $i \neq r$. Thus $T_{i, j_{i}}, 1 \leq i \leq \ell, 1 \leq j_{i} \leq$ $k_{i}-k_{i-1}+1$ are $k+\ell$ internally disjoint $S$-trees.

We now show that bounds of Theorem 1.5 are sharp. For $(i)$, Example 3.1 is a sharp example. Let $K_{n}$ be a complete graph with $n$ vertices, and $P_{m}$ be a path with $m$ vertices, where $m \geq 2$. We have $\kappa_{3}\left(P_{m}\right)=1$, and $\kappa_{3}\left(K_{n}\right)=n-2$ by Theorem 1.2. It is easy to check that $\kappa_{3}\left(K_{n} \square P_{m}\right)=n-2+1=n-1$. Thus, $K_{n} \square P_{m}$ is a sharp example for $(i i)$.

\section{Acknowledgments}

We thank anonymous reviewers for their carefully reading of our work and their helpful suggestions.

\section{References}

[1] J.A. Bondy, U.S.R. Murty, Graph Theory, GTM 244, Springer, 2008.

[2] G. Chartrand, S.F. Kapoor, L.Lesniak, D.R. Lick, Generalized connectivity in graphs, Bull. Bombay Math. Colloq. 2(1984), 1-6

[3] G. Chartrand, F. Okamoto, P. Zhang, Rainbow trees in graphs and generalized connectivity, Networks 55(4)(2010), 360-367.

[4] W.S. Chiue, B.S. Shieh, On connectivity of the Cartesian product of two graphs. Appl. Math. Comput. 102(1999), 129-137. 
[5] W. Imrich, S. Klavžar, Product Graphs Structure and Recongnition, A Wiley-Interscience Publication, 2000.

[6] S. Klavžar, S. Špacapan, On the edge-connectivity of Cartesian product graphs, Asian-Eur. J. Math. 1(2008), 93-98.

[7] S. Li, W. Li, X. Li, The generalized connectivity of complete bipartite graphs, Ars Combin. 104(2012).

[8] S. Li, X. Li, Note on the hardness of generalized connectivity, J. Comb. Optim., doi 10.1007/s10878011-9399-x.

[9] S. Li, X. Li, W. Zhou, Sharp bounds for the generalized connectivity $\kappa_{3}(G)$, Discrete Math. 310(2010), 2147-2163.

[10] F. Okamoto, P. Zhang, The tree connectivity of regular complete bipartite graphs, J. Combin. Math. Combin. Comput. 74(2010), 279-293.

[11] G. Sabidussi, Graphs with given group and given graph theoretical properties, Canadian J. Math. 9(1957), 515-525.

[12] S. Špacapan, Connectivity of Cartesian products of graphs. Appl. Math. Lett. 21(2008), 682-685.

[13] N.A. Sherwani, Algorithms for VLSI physical design automation, 3rd Edition, Kluwer Acad. Pub., London, 1999.

[14] H. Whitney, Congruent graphs and the connectivity of graphs, Am. J. Math. 54(1932), 150-168.

[15] I. Wilfried, S. Klavžar, D.F. Rall, Topics in Graph Theory. Graphs and Their Cartesian Product. A K Peters, 2008.

[16] J.M. Xu, C. Yang, Connectivity of Cartesian product graphs. Discrete Math. 306(1)(2006), 159-165 